\title{
Intravenous Thrombolysis for Suspected Ischemic Stroke with Seizure at Onset
}

Alexandros A. Polymeris, $\mathrm{MD}^{1}$; Sami Curtze, MD, MSc, $\mathrm{PhD}^{2}$; Hebun Erdur, $\mathrm{MD}^{3}$; Christian Hametner, $\mathrm{MD}^{4}$; Mirjam R. Heldner, MD, MSc${ }^{5}$; Adrien E. Groot, MD ; Andrea Zini, MD; Yannick Béjot, MD, $\mathrm{PhD}^{8}$; Annina Dietrich ${ }^{9}$; Nicolas Martinez-Majander, MD, MSc ${ }^{2}$; Regina von Rennenberg, $\mathrm{MD}^{3}$; Christoph Gumbinger, $\mathrm{MD}^{4}$; Sabine Schaedelin, $\mathrm{MSc}^{10}$; Gian Marco De Marchis, MD, MSc ${ }^{1}$; Sebastian Thilemann, MD ${ }^{1}$; Christopher Traenka, MD ${ }^{1,11}$; Philippe A.

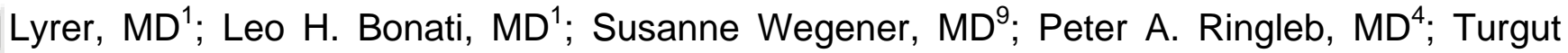
Tatlisumak, MD, $\mathrm{PhD}^{2,12}$; Christian H. Nolte, $\mathrm{MD}^{3}$; Jan F. Scheitz, $\mathrm{MD}^{3}$; Marcel Arnold, $\mathrm{MD}^{5}$; Daniel Strbian, MD, MSc, PhD², Paul J. Nederkoorn, MD, PhD ${ }^{6}$; Henrik Gensicke, MD ${ }^{1,11}$ and Stefan T. Engelter, $\mathrm{MD}^{1,11}$ for the TRISP Collaborators

${ }^{1}$ Department of Neurology and Stroke Center, University Hospital Basel and University of Basel, Basel, Switzerland

Department of Neurology, Charité-Universitaetsmedizin Berlin, Berlin, Germany

${ }^{4}$ Department of Neurology, University Hospital Heidelberg, Heidelberg, Germany

${ }^{5}$ Department of Neurology, Inselspital, Bern University Hospital and University of Bern, Bern,

Switzerland

${ }^{6}$ Department of Neurology, Amsterdam UMC, University of Amsterdam, Amsterdam, the Netherlands

${ }^{7}$ IRCCS Istituto di Scienze Neurologiche di Bologna, Department of Neurology and Stroke Center, Maggiore Hospital, Bologna, Italy

${ }^{8}$ University Hospital and Medical School of Dijon, University of Burgundy, France

${ }^{9}$ Department of Neurology, University Hospital and University of Zurich, Zurich, Switzerland

${ }^{10}$ Clinical Trial Unit, Department of Clinical Research, University Hospital Basel and University of Basel, Basel, Switzerland

${ }^{11}$ Veurology and Neurorehabilitation, University Department of Geriatric Medicine Felix Platter; University of Basel, Basel, Switzerland

${ }^{12}$ Department of Neurology, Sahlgrenska University Hospital and Department of Clinical Neuroscience, Institute of Neuroscience and Physiology, Sahlgrenska Academy at University of Gothenburg, Gothenburg, Sweden 


\section{Correspondence to:}

Alexandros A. Polymeris

Department of Neurology and Stroke Center

University Hospital Basel and University of Basel

Petersgraben 4, 4031 Basel, Switzerland

Alexandros.Polymeris@usb.ch

Running Head: IVT for Suspected Stroke with Seizure at Onset

Keywords: intravenous thrombolysis, seizure at onset, treatment outcome

Characters in Title: 76

Characters in Running Head: 46

Word Count Abstract: 244/250

Word Count Manuscript: 4,094/4,500 (Introduction: 395/500, Discussion: 986/1,500)

References: 46/50

Figures: 1

Tables: 2, Supplemental tables: 5 


\section{Abstract}

\section{Objective}

Seizure at onset ( $\mathrm{SaO}$ ) has been considered a relative contraindication for intravenous thrombolysis (IVT) in patients with acute ischemic stroke, although this appraisal is not evidence-based. Here, we investigated the prognostic significance of $\mathrm{SaO}$ in patients treated with IVT for suspected ischemic stroke.

\section{Methods}

In this multicenter, IVT-registry-based study we assessed the association between SaO and symptomatic intracranial hemorrhage ( $\mathrm{SICH}$, ECASS-II definition), 3-month mortality and 3month functional outcome on the modified Rankin Scale (mRS) using unadjusted and adjusted logistic regression, coarsened exact matching and inverse probability weighted analyses.

\section{Results}

Among 10,074 IVT-treated patients, 146 (1.5\%) had SaO. SaO patients had significantly higher NIHSS and glucose on admission and more often female sex, prior stroke and prior functional dependence than non-SaO patients. In unadjusted analysis, they had generally less favorable outcomes. After controlling for confounders in adjusted, matched and weighted analyses, all associations between $\mathrm{SaO}$ and any of the outcomes disappeared, including $\mathrm{sICH}$ (odds ratio [OR] $]_{\text {unadjusted }} 1.53$ [95\% confidence interval: 0.74,3.14], OR $\mathrm{R}_{\text {adjusted }}$ $\left.0.52[0.13,2.16], \mathrm{OR}_{\text {matched }} 0.68[0.15,3.03], \mathrm{OR}_{\text {weighted }} 0.95[0.39,2.32]\right)$, mortality (OR unadjusted $1.49[1.00,2.24], \mathrm{OR}_{\text {adjusted }} 0.98[0.5,1.92], \mathrm{OR}_{\text {matched }} 1.13[0.55,2.33]$, OR $\left.\mathrm{R}_{\text {weighted }} 1.17[0.73,1.88]\right)$ and functional outcome (mRS $\geq 3$ / ordinal mRS: OR unadjusted 1.33[0.96,1.84] / 1.35[1.01,1.81], $\mathrm{OR}_{\text {adjusted }} 0.78[0.45,1.32] / 0.78[0.52,1.16], \mathrm{OR}_{\text {matched }} 0.75[0.43,1.32] / 0.45[0.10,2.06]$, $\left.\sim R_{\text {weighted }} 0.87[0.57,1.34] / 1.00[0.66,1.52]\right)$. These results were consistent regardless of whether patients had an eventual diagnosis of ischemic stroke (89/146) or stroke mimic (57/146 SaO patients).

\section{Interpretation}

$\mathrm{SaO}$ was not an independent predictor of poor prognosis. Withholding IVT from patients with assumed ischemic stroke presenting with $\mathrm{SaO}$ seems unjustified. 


\section{Introduction}

Intravenous thrombolysis (IVT) is effective and safe in selected patients with acute ischemic stroke. ${ }^{1}$ A seizure at onset ( $\left.\mathrm{SaO}\right)$ of an acute stroke syndrome is uncommon, but has traditionally been considered as a relative contraindication for IVT. ${ }^{2-4}$

This traces back to the explicit exclusion of patients with SaO from the pivotal National Institute of Neurological Disorders and Stroke (NINDS) randomized controlled trial ${ }^{5}$, as well as from the vast majority of subsequent IVT trials for acute ischemic stroke ${ }^{1}$. The rationale for this was that neurological deficits associated with seizures usually reflect a transient postictal phenomenon mimicking a stroke and that such patients should not be exposed to an unnecessary bleeding risk. ${ }^{4}$ However, seizures do also occur at the onset of an actual acute ischemic stroke. ${ }^{6}$ Since such patients were excluded from the trials, there is currently only weak evidence for the use of IVT in stroke patients with SaO.

The latest AHA/ASA Guidelines (2018) removed SaO from the IVT contraindications list and make a moderate recommendation for IVT in patients with $\mathrm{SaO}$ "if evidence suggests that residual impairments are secondary to stroke and not a postictal phenomenon". ${ }^{7}$ However, differentiating between these two entities in the acute phase, when time is precious, is challenging, especially in the absence of advanced imaging. ${ }^{8-11}$ Observational data suggest that IVT seems to be safe in patients with stroke mimics, of whom several had had seizures with postictal symptoms but without acute stroke. ${ }^{12-15}$ As these diagnoses were usually made retrospectively, the applicability in the acute management of patients presenting with $\mathrm{SaO}$ is limited. Furthermore, the prognostic importance of a seizure at presentation is also unclear among those IVT-treated patients who eventually had a verified acute ischemic stroke. For stroke physicians considering IVT in patients with suspected ischemic stroke and SaO, more data about safety and effectiveness are needed to inform their decisions in the acute phase.

With these considerations in mind, we investigated the prognostic significance of SaO in patients treated with IVT for assumed ischemic stroke. This included both patients who were eventually diagnosed with an acute stroke and those with a final diagnosis of a stroke mimic. Reflecting clinical practice, we analyzed both groups together in the main analysis. In ancillary analyses we assessed for interaction between final diagnosis and prognostic significance of $\mathrm{SaO}$ and repeated all analyses in the subgroup of patients with eventual diagnosis of ischemic stroke. 


\section{Methods}

\section{Study design, patient population and data collection}

For this study, we pooled data on prospectively registered, consecutive patients with suspected acute ischemic stroke treated with IVT alone or IVT followed by endovascular treatment (EVT) from 9 European stroke centers within the ThRombolysis in Ischemic Stroke Patients (TRISP) Collaboration. The detailed methodology of TRISP has been previously published. ${ }^{16}$ In short, data were collected locally at each TRISP center using a standardized form with predefined parameters and were pooled and analyzed at the TRISP center Basel.

This study had been submitted to the ethics committee in the leading center Basel. The committee had no ethical concerns and waived the necessity to obtain informed consent from individual patients (Ethics Committee of Northwest and Central Switzerland UBE-15/118). The requirement for local ethics approval differed between participating centers, and approval was obtained if required. The list of TRISP centers participating in this study including number of patients contributed by each center and time period of data collection, as well as the complete list of all TRISP centers and collaborators are given in Supplemental Table 1.

The main parameter of interest for this study was presence versus absence of SaO. SaO was defined adapting previously reported definitions ${ }^{6,17}$ as any clinically evident seizure, as observed by witnesses or medical providers, from stroke onset to IVT (i.e., not later than the end of the IVT infusion). Patients with missing information on presence or absence of SaO were excluded from the study.

The following baseline TRISP parameters were used: age, sex, vascular risk factors according to predefined criteria ${ }^{16}$, prior stroke, prior antithrombotic treatment (antiplatelets and/or anticoagulants), prior functional dependence (defined as pre-stroke modified Rankin srale $(m R S) \geq 3$ ), National Institutes of Health Stroke Scale (NIHSS) score on admission, estimated glomerular filtration rate (eGFR) according to the Chronic Kidney Disease Epidemiology Collaboration formula ${ }^{18}$ and glucose on admission, onset-to-treatment time, treatment with IVT alone or IVT followed by EVT, presence or absence of early hypodensity on initial computed tomography (CT) scan (as in prior research ${ }^{18}$, added post hoc) and final diagnosis of ischemic stroke or stroke mimic. Stroke mimics were labeled as such when any final diagnosis other than stroke was made by the patients' treating physicians after exclusion of an ischemic cause of the stroke-like symptoms based on clinical examinations and neuroimaging findings, as previously described. ${ }^{12,14}$ 
Outcome measures were: (i) symptomatic intracranial hemorrhage $(\mathrm{sICH})$, according to the European Cooperative-Acute-Stroke-Study-II (ECASS-II) definition ${ }^{19}$, (ii) all-cause mortality at 3 months and (iii) functional outcome on the mRS at 3 months. As previously described ${ }^{16}$, the 3-month outcomes were assessed during outpatient visits or with postal questionnaires or telephone calls with patients, patients' relatives and/or general practitioners as applicable.

For the SaO patients we reviewed the detailed medical charts post hoc to provide a full clinical and radiological characterization of these cases. We systematically collected the following parameters: type of seizure (according to the 2017 International League Against Epilepsy classification ${ }^{20}$ ), timing of seizure with regards to hospital admission and IVT, seizure duration, treatment of the seizure, seizure recurrence during hospital stay, information on neuroimaging modalities used before IVT and thereafter and neuroimaging findings (including $\mathrm{SICH}$ according to the ECASS-III definition ${ }^{21}$ ), presence or absence of potentially confounding factors ${ }^{22,23}$ including electrolyte abnormalities, hypoglycemia, chronic ischemic brain lesions, intracranial tumors, brain vascular malformations, meningoencephalitis, endocarditis, cerebral venous thrombosis, history of traumatic brain injury or intracranial hemorrhage, alcohol or drug abuse, history of seizures or epilepsy, prior use of antiepileptic drugs and benzodiazepines, dementia or other neurodegenerative disease, multiple sclerosis or other neuroinflammatory disease and psychiatric or neurodevelopmental disorders. For the $\mathrm{SaO}$ patients with a final diagnosis of stroke mimic we also collected the cause to which the seizures were finally attributed.

\section{Statistical analyses}

We used descriptive statistics to present the baseline data and the outcome measures. For continuous variables, the mean and standard deviation (SD) or, in case of skewed data, the median and interquartile range (IQR) were used. We present categorical variables as count and frequency. We report the rate of missing values for all variables.

We compared baseline data between patient subgroups using the $x^{2}$ test for categorical variables and the t-test or Mann-Whitney $U$ test as appropriate for continuous variables. A $p$ value $<0.05$ was considered statistically significant.

To assess the association between $\mathrm{SaO}$ and the respective outcome measures we used the following methods:

(i) Unadjusted logistic regression

(ii) Logistic regression adjusted for confounding by the following potentially outcomemodifying parameters, as in prior research ${ }^{18,24}$ : age, sex, NIHSS on admission, prior 
antithrombotic treatment, glucose and eGFR on admission, EVT in addition to IVT, onsetto-treatment time, prior dependence and prior stroke. Missing values in these variables were imputed using five multiple imputations according to van Buuren and GroothuisOudshoorn. ${ }^{25}$

(iii) Matched analysis using coarsened exact matching. With this monotonic imbalance bounding matching method, ${ }^{26}$ patients with and without SaO were matched using all patients who had at least one matching partner. When several patients qualified as matching partners, they were weighted accordingly to avoid overrepresentation. In the matching model, the number of variables was reduced to the variables with the most imbalance in order to maintain enough matches. Thus, the variables age, sex, NIHSS, glucose and eGFR on admission, prior stroke and final diagnosis of ischemic stroke or stroke mimic were included in the model. For the matching process, continuous variables were converted to categorical using 15 cut-off points according to Sturges' rule. In case of missing values, an additional category labeled "missing values" was introduced in the variables included in the matching model. The matched data were then fit in a univariable logistic model as in (i).

(iv) Inverse probability weighted analysis. For this analysis, the conditional probability of SaO was estimated for each patient using a logistic model with $\mathrm{SaO}$ as the outcome variable and the following parameters as independent variables: age, sex, NIHSS on admission, prior antithrombotic treatment, glucose and eGFR on admission, EVT in addition to IVT, onset-to-treatment time, prior stroke and final diagnosis of ischemic stroke or stroke mimic. The inverse probability weight (IPW $)^{27,28}$ was then calculated for each patient, and by multiplying the IPW by the unconditional probability of SaO (derived by a logistic model with $\mathrm{SaO}$ as outcome and an intercept only, without covariates ${ }^{28,29}$ ), the stabilized IPW was calculated. The stabilized IPW was finally included in the adjusted logistic model described in (ii), and robust standard errors were used for the confidence intervals of this weighted analysis. In the models used to estimate the IPW, as well as in the weighted logistic model, missing values for the covariates were imputed using single imputation. Thereby the mean was imputed for continuous variables and the mode (i.e., the most common category) for categorical variables.

For each method (i) - (iv), the model-based odds ratio (OR) estimate, 95\% confidence interval $(\mathrm{Cl})$ and $\mathrm{p}$-value are presented.

To investigate potential differences in the prognostic significance of $\mathrm{SaO}$ in patients with a final diagnosis of ischemic stroke compared to those with a final diagnosis of stroke mimic, we performed the following ancillary analyses: 
(a) We repeated the adjusted logistic regression analyses described in (ii) with the addition of the covariate "final diagnosis" and the interaction term "SaO * final diagnosis" in the model.

(b) We repeated all analyses (i) - (iv) described above in the subgroup of patients with a final diagnosis of ischemic stroke (thereby excluding patients with a final diagnosis of stroke mimic and without using the covariate final diagnosis in these models). Repeating the analyses in the subgroup of patients with a final diagnosis of a stroke mimic was not possible due to the low number of patients and outcomes.

The analyses described above were performed for all study outcomes including $\mathrm{SICH}$, mortality at 3 months and mRS at 3 months both as a dichotomized binary variable (defining poor functional outcome as $\mathrm{mRS} \geq 3$ ) and as an ordinal variable with 7 levels (mRS $0-6$ ). For the latter, the analyses were performed using proportional odds logistic models (i.e., shift models). Finally, as an ancillary analysis we repeated the unadjusted and adjusted logistic regression analyses (i) and (ii) for the outcome mRS on survivors only, after excluding patients with $\mathrm{mRS}=6$. Missing values in the outcome measures were imputed using five multiple imputations ${ }^{25}$ for the unadjusted and adjusted logistic regression analyses (i) and (ii), but not for the matched and weighted analyses (iii) and (iv).

All analyses were performed in $\mathrm{R}$ version 3.5.0 (Foundation for Statistical Computing, Vienna, Austria). 


\section{Results}

Out of 10,105 patients treated with IVT over a period spanning from 1998 to 2017 across the participating TRISP centers, information on SaO was missing in $31(0.3 \%)$, yielding a total of 10,074 patients eligible for analysis (median [IQR] age 73 [62 - 80] years, 44.7\% female, 10.3\% IVT followed by EVT).

Among study patients, $146(1.5 \%)$ had SaO. SaO patients had significantly higher NIHSS scores (median [IQR] 13 [7 - 21] vs 9 [5 - 15], $p<0.001$ ) and glucose levels on admission (median [IQR] 7.1 [6.1 - 8.5] vs 6.6 [5.8 - 8.0], $p=0.016)$, were more often female ( $54.1 \%$ vs $44.6 \%, p=0.027)$, had more often prior stroke $(25.7 \%$ vs $16.5 \%, p=0.005)$ and prior functional dependence $(16.4 \%$ vs $7.6 \%, p<0.001)$ and more often final diagnosis of a stroke mimic (39\% vs $2.1 \%, p<0.001)$ than patients without SaO. Patients' baseline characteristics stratified to presence or absence of $\mathrm{SaO}$ are summarized in Table 1.

\section{SICH}

Among SaO patients, 8/146 (5.7\%) had sICH compared to 359/9,806 (3.7\%) in non-SaO patients. After adjustment for the aforementioned outcome-modifying variables, there was no difference in the odds for $\mathrm{SICH}$ between $\mathrm{SaO}$ and non-SaO patients in the logistic model $\left(\mathrm{OR}_{\text {adjusted }} 0.52,95 \% \mathrm{Cl}[0.13,2.16]\right)$. The same was true in the matched $\left(\mathrm{OR}_{\text {matched }} 0.68\right.$, $95 \% \mathrm{Cl}[0.15,3.03])$ and weighted analysis (OR $\left.\mathrm{OR}_{\text {weighted }} 0.95,95 \% \mathrm{Cl}[0.39,2.32]\right)$.

\section{All-cause mortality at $\mathbf{3}$ months}

At 3 months, 30/146 $\mathrm{SaO}$ patients (20.5\%) had died compared to 1,432/9,709 non-SaO patients (14.8\%). After adjustment for confounders, there was no difference in the odds for 3month mortality between $\mathrm{SaO}$ and non-SaO patients in the logistic model (OR adjusted 0.98, $95 \% \mathrm{Cl}[0.5,1.92]$. This held true in the matched $\left(\mathrm{OR}_{\text {matched }} 1.13,95 \% \mathrm{Cl}[0.55,2.33]\right)$ and weighted analysis ( $\left.\mathrm{OR}_{\text {weighted }} 1.17,95 \% \mathrm{Cl}[0.73,1.88]\right)$.

\section{. . Inctional outcome on the $\mathrm{mRS}$ at 3 months}

At 3 months, 75/146 SaO patients (51.4\%) had poor functional outcome (mRS $\geq 3$ ) compared to 4,277/9,656 non-SaO patients (44.3\%). After adjustment for confounders, the odds for poor functional outcome did not differ between $\mathrm{SaO}$ and non-SaO patients in the logistic model $\left(\mathrm{OR}_{\text {adjusted }} 0.78,95 \% \mathrm{Cl}[0.45,1.32]\right)$, nor in the matched $\left(\mathrm{OR}_{\text {matched }} 0.75,95 \% \mathrm{Cl}\right.$ $[0.43,1.32])$ and weighted analysis $\left.\mathrm{OR}_{\text {weighted }} 0.87,95 \% \mathrm{Cl}[0.57,1.34]\right)$. Similarly, when examining $\mathrm{mRS}$ as an ordinal variable, the adjusted, matched and weighted analyses showed no difference in the distribution of the mRS scores between $\mathrm{SaO}$ and non-SaO patients $\left(\mathrm{OR}_{\text {adjusted }} 0.78,95 \% \mathrm{Cl}[0.52,1.16]\right.$; $\mathrm{OR}_{\text {matched }} 0.45,95 \% \mathrm{Cl}[0.10,2.06]$; OR $\mathrm{R}_{\text {weighted }}$ 
$1.00,95 \% \mathrm{Cl}[0.66,1.52])$. Consistent with the main analysis, there was no association between $\mathrm{SaO}$ and 3-month $\mathrm{mRS}$ in the adjusted logistic models after including only survivors.

Figure 1 shows the odds ratio estimates for $\mathrm{sICH}, 3$-month mortality, poor 3-month functional outcome ( $m R S \geq 3$ ) and shift to a higher 3-month $\mathrm{mRS}$ in patients with versus without SaO in the overall study population. The detailed results of the main analysis are presented in Table 2. The detailed results of the mRS analyses in survivors only are given in Supplemental Table 2. Supplemental Table 3 descriptively presents the outcome measures stratified to presence or absence of SaO separately in patients treated with IVT alone and those treated with IVT followed by EVT.

\section{Interaction between final diagnosis and prognostic significance of SaO}

Ancillary analyses in the adjusted logistic models revealed no interaction effect between SaO and final diagnosis on the outcomes $\mathrm{sICH}(p=0.97$ for interaction) and 3-month mRS, the latter both as a dichotomized $(p=0.72)$ and as an ordinal variable $(p=0.80)$ and also after including only survivors ( $p=0.31$ and 0.26 respectively). The ancillary analysis for interaction between $\mathrm{SaO}$ and final diagnosis was not performed for 3-month mortality because the low number of patients with stroke mimics and death did not allow for a stable estimate of the interaction term.

\section{Subgroup analysis in patients with final diagnosis of ischemic stroke}

In the subgroup of 9,612 patients with a final diagnosis of ischemic stroke, sICH occurred in $5 / 89$ SaO patients (5.6\%) and in 345/9,421 non-SaO patients (3.7\%). Death at 3 months was reported in 28/89 SaO patients (31.5\%) compared to 1,388/9,325 non-SaO patients (14.9\%). Poor 3-month functional outcome was observed in 50/89 SaO patients (56.2\%) compared to $4,160 / 9,285$ non-SaO patients (44.8\%). Consistent with the main analysis, the ancillary adjusted, matched and weighted analyses revealed no significant differences between SaO and non-SaO patients with final diagnosis of ischemic stroke in any of the outcomes. Details ure given in Supplemental Table 2.

\section{Clinical and neuroimaging characterization of the SaO patients}

Among the 146 patients with $\mathrm{SaO}$, the seizure had focal onset without impaired awareness in 14 patients (9.6\%), focal onset with impaired awareness in $37(25.3 \%)$ and focal onset with evolution to bilateral tonic-clonic seizure or generalized onset in $81(55 \%)$. The SaO had occurred before hospital admission in 111 patients (76\%), after admission and before IVT in $18(12.3 \%)$ and during IVT in $17(11.6 \%)$. Acute antiepileptic treatment was administered to 65 patients (45.8\%), and 73 patients (50\%) were prescribed long-term antiepileptic treatment. Seizures recurred during hospital stay in 28 patients (19.6\%). Further details are given in 
Supplemental Table 4. Most common potentially confounding factors in SaO patients were chronic ischemic brain lesions in 30 patients (20.5\%) and prior use of antiepileptic drugs and/or benzodiazepines in $28(19.2 \%)$. The initial neuroimaging modality was CT in 121 patients (82.9\%), magnetic resonance imaging (MRI) in $21(14.4 \%)$ and both in $4(2.7 \%)$. The final diagnosis was stroke mimic in 6/25 (24\%) SaO patients that had been initially evaluated with MRI (with or without CT) compared to 51/121 (42.1\%) SaO patients that were initially evaluated with CT alone $(p=0.09)$. The final diagnosis was stroke mimic in 32/106 (30.2\%) $\mathrm{SaO}$ patients that had initially been evaluated with advanced imaging (angiography with or without perfusion) compared to 25/40 (62.5\%) SaO patients without initial advanced imaging $(p<0.001)$. Among SaO patients with a final diagnosis of stroke mimic, 44/57 (77.2\%) had a clear cause of the seizure (details in Supplemental Table 5). 


\section{Discussion}

This large, observational, multicenter study on the prognostic impact of seizure at onset in patients treated with IVT for assumed ischemic stroke showed the following key findings: (i) $\mathrm{SaO}$ patients had a more unfavorable baseline risk profile compared to non-SaO patients, including higher stroke severity and more often prior stroke and pre-existing dependence. (ii) After controlling for confounders, $\mathrm{SaO}$ was not an independent predictor for $\mathrm{SICH}$, nor for mortality and functional outcome at 3 months. (iii) Results were consistent regardless of whether patients were eventually diagnosed with an ischemic stroke or a stroke mimic.

Whether to use or refrain from IVT in SaO patients is controversial, as there is currently hardly any evidence which would allow balancing risks versus benefit of IVT in these patients. With this study we provide data indicating that in patients treated with IVT for assumed ischemic stroke, functional outcome as well as the odds for complications including $\mathrm{sICH}$ and mortality are not modified by the presence or absence of SaO. In the absence of randomized controlled data on this issue, our results might be clinically important for individual treatment decisions.

Until now, data on SaO patients were included in reports about the prognostic significance of early seizures, which were defined as seizures occurring within 7 days $^{30}, 72$ hours $^{31}$ and 24 hours $^{32}$ after stroke onset, respectively. According to all these reports, early seizures indicated a poor prognosis. ${ }^{30-32}$ However, SaO-patients were only a small minority, accounting for 3 out of $>3,000$ IVT-treated patients in the ENCHANTED trial ${ }^{30}, 1$ out of 12 thrombolyzed stroke patients with an early seizure ${ }^{31}$ and 7 out of 800 stroke patients receiving endovascular therapy ${ }^{32}$.

Thus, putting our observations in the context of the aforementioned studies, SaO should be distinguished from early seizures, as they seem to differ with regards to prognosis after IVTtreatment. Interestingly, Rodan et al reported on 3 stroke patients who developed a seizure during IVT administration followed by dramatic neurological recovery and postulated that seizure was a favorable prognostic sign, possibly arising due to cortical reperfusion or hyperperfusion after recanalization of an occluded intracranial artery. ${ }^{33}$

In our study, SaO patients had higher NIHSS and blood glucose on admission and more often prior stroke and prior functional dependence compared to non-SaO patients, factors which are known to be associated with worse prognosis. ${ }^{34-37}$ Indeed, SaO patients had a higher rate of $\mathrm{sICH}$, death and poor functional outcome. However, the fact that SaO did not independently predict poor outcome after accounting for these confounders indicates that it 
should be considered as a marker for an unfavorable risk profile rather than a predictor for poor outcome per se. This is in line with previous reports on early post-stroke seizures being associated with unfavorable baseline risk factors, including higher stroke severity ${ }^{30}$, 32, 38-42 and higher admission blood glucose ${ }^{40,41}$. Larger, non-lacunar supratentorial infarcts with cortical involvement ${ }^{31}, 38,39,41-43$ and a potential neurotoxic, epileptogenic effect of hyperglycemia ${ }^{40,41}$ might explain these associations. Furthermore, the higher rate of prior stroke and prior functional dependence in SaO patients raises the possibility of post-stroke seizures. Indeed, structural post-stroke epilepsy was the most common cause of seizures in $\mathrm{SaO}$ patients with eventual diagnosis of a stroke mimic.

Our observation that a final diagnosis of stroke mimic does not modify our key findings confirms and refines reports about the relative safety of IVT in patients with stroke mimics, including patients with (mostly retrospectively diagnosed) seizures ${ }^{12-15}$. We observed a lower rate of stroke mimics in SaO patients that were initially evaluated with advanced imaging and $\mathrm{MRI}$, pointing to the usefulness of these modalities in differentiating stroke from stroke mimics. $8-11,44,45$

As a novelty, we observed that also among IVT-treated patients with an eventual diagnosis of ischemic stroke, SaO had no independent impact on prognosis. This is important, as it advances the so far limited level of evidence and might therefore increase the strength of the currently moderate AHA/ASA recommendation ${ }^{7}$ for IVT in such patients.

The strengths of our study involving 9 European stroke centers with long-standing experience in IVT include (i) its rigorous statistical methodology, employing 3 distinct methods to control for confounders that all yielded consistent results, (ii) its large sample size, which allowed for adjustment for several confounding factors and which reduces the risk of spurious findings, (iii) the high rate of data completeness, reducing the risk of bias, (iv) the prospective and ctandardized data collection on the outcomes and ( $v$ ) its multicenter design, indicating a certain generalizability of our results, at least within the Caucasian patient population treated in experienced stroke centers.

However, the following potential limitations need to be acknowledged: (i) The TRISP collaborative registry is not monitored and outcomes are not adjudicated. (ii) As TRISP includes only IVT-treated patients, we did not have a comparison group of patients with SaO not treated with IVT. (iii) We do not know in how many SaO patients, otherwise eligible for IVT, this treatment was withheld because of the SaO. The fact that our rate of SaO among IVT-treated patients (i.e.,1.5\%) was lower than that of "seizures in the acute stroke setting" 
reported elsewhere (i.e., 3.6\% among 461 patients), indicates that this might have happened, although the definition of seizures in that study was unclear ${ }^{46}$ and although another study using a definition similar to ours reported a lower rate of seizure at presentation (i.e., $0.16 \%$ among 20,000 ischemic stroke patients). ${ }^{6}$ (iv) Patients with unwitnessed stroke onset might have had an unobserved seizure and might therefore have been misclassified as non-SaO.

(v) Due to the structure of data retrieval in TRISP, data on the semiology of SaO and history of epilepsy had to be added post hoc after review of the patients' medical charts.

In conclusion, among patients treated with IVT for suspected ischemic stroke, seizure at onset was not an independent predictor of poor prognosis. Withholding IVT from otherwise eligible patients with seizure at the onset of an acute stroke syndrome seems unjustified.

\section{Acknowledgments}

AAP has received support from the Swiss Academy of Medical Sciences and the BangerterFoundation (YTCR 31/17). This study has been supported by the Wissenschaftspool, Medical Division of the University Hospital Basel, Switzerland.

\section{Author contributions}

AAP and STE conceived and designed the study, with additional input from SC, $\mathrm{HE}, \mathrm{CH}, \mathrm{AZ}$, YB, GMDM, PAL, SW, PAR, TT, CHN, JFS, MA, DS, PJN and HG. All authors contributed to data acquisition, analysis and interpretation. AAP, SS and STE drafted the manuscript. The members of the TRISP Collaboration and their institutional affiliations are given in Supplemental Table 1.

\section{Conflicts of interest}

$A Z$ has received speaker honoraria, consulting fees and advisory board compensation from Boehringer-Ingelheim (the producer of Actilyse ${ }^{\circledR}$ ), speaker honoraria and consulting fees from Medtronic and advisory board compensation from Stryker (both producers of revascularization devices for acute ischemic stroke). YB has received honoraria or consulting fees from Medtronic and Boehringer-Ingelheim. AD has been supported by a BoehringerIngelheim research grant. PAL has received travel and speaker honoraria, advisory board compensation and research funding from Boehringer-Ingelheim. SW has been supported by a Boehringer-Ingelheim research grant. PAR has received lecture fees, travel and advisory board compensation and research funding (for the ECASS4 study) from Boehringer- 
Ingelheim and travel compensation from SITS. TT has had research contracts with Boehringer-Ingelheim, has received advisory board compensation from Boehringer-Ingelheim and Lumosa Pharmaceuticals (developer of novel medical therapies for acute stroke), and holds a patent on pharmacological treatment of post-thrombolytic brain hemorrhage and brain edema. $\mathrm{CHN}$ has received lecture and consultancy fees from Boehringer-Ingelheim. MA has received speaker and advisory board honoraria from Boehringer-Ingelheim and Covidien (producer of revascularization devices for acute ischemic stroke). PJN has received consulting fees from Boehringer-Ingelheim. STE has received travel or speaker honoraria from Boehringer-Ingelheim and advisory board compensation from Boehringer-Ingelheim and Covidien. The rest of the authors have no conflicts of interest to declare. 


\section{References}

1. Emberson J, Lees KR, Lyden P, et al. Effect of treatment delay, age, and stroke severity on the effects of intravenous thrombolysis with alteplase for acute ischaemic stroke: a meta-analysis of individual patient data from randomised trials. Lancet (London, England). 2014 Nov 29;384(9958):1929-35.

2. Adams HP, Jr., del Zoppo G, Alberts MJ, et al. Guidelines for the early management of adults with ischemic stroke: a guideline from the American Heart Association/American Stroke Association Stroke Council, Clinical Cardiology Council, Cardiovascular Radiology and Intervention Council, and the Atherosclerotic Peripheral Vascular Disease and Quality of Care Outcomes in Research Interdisciplinary Working Groups: The American Academy of Neurology affirms the value of this guideline as an educational tool for neurologists. Circulation. 2007 May 22;115(20):e478-534.

3. Jauch EC, Saver JL, Adams HP, Jr., et al. Guidelines for the early management of patients with acute ischemic stroke: a guideline for healthcare professionals from the American Heart Association/American Stroke Association. Stroke. 2013 Mar;44(3):870-947.

4. Demaerschalk BM, Kleindorfer DO, Adeoye OM, et al. Scientific Rationale for the Inclusion and Exclusion Criteria for Intravenous Alteplase in Acute Ischemic Stroke: A Statement for Healthcare Professionals From the American Heart Association/American Stroke Association. Stroke. 2016 Feb;47(2):581-641.

5. Tissue Plasminogen Activator for Acute Ischemic Stroke. New England Journal of Medicine. 1995;333(24):1581-8.

6. Cheng JH, Zhang Z, Ye Q, Ye ZS, Xia NG. Characteristics of the ischemic stroke patients whose seizures occur at stroke presentation at a single institution in Eastern China. J Neurol Sci. 2018 Apr 15;387:46-50.

7. Powers WJ, Rabinstein AA, Ackerson T, et al. 2018 Guidelines for the Early Management of Patients With Acute Ischemic Stroke: A Guideline for Healthcare Professionals From the American Heart Association/American Stroke Association. Stroke. 2018 Mar;49(3):e46-e110.

8. Masterson K, Vargas MI, Delavelle J. Postictal deficit mimicking stroke: role of perfusion CT. Journal of neuroradiology Journal de neuroradiologie. 2009 Mar;36(1):48-51.

э. Kubiak-Balcerewicz K, Fiszer U, Naganska E, et al. Differentiating Stroke and Seizure in Acute Setting-Perfusion Computed Tomography? Journal of stroke and cerebrovascular diseases : the official journal of National Stroke Association. 2017 Jun;26(6):1321-7.

10. Sylaja PN, Dzialowski I, Krol A, Roy J, Federico P, Demchuk AM. Role of CT angiography in thrombolysis decision-making for patients with presumed seizure at stroke onset. Stroke. 2006 Mar;37(3):915-7.

11. Selim M, Kumar S, Fink J, Schlaug G, Caplan LR, Linfante I. Seizure at stroke onset: should it be an absolute contraindication to thrombolysis? Cerebrovascular diseases (Basel, Switzerland). 2002;14(1):54-7. 
12. Winkler DT, Fluri F, Fuhr P, et al. Thrombolysis in stroke mimics: frequency, clinical characteristics, and outcome. Stroke. 2009 Apr;40(4):1522-5.

13. Chernyshev OY, Martin-Schild S, Albright KC, et al. Safety of tPA in stroke mimics and neuroimaging-negative cerebral ischemia. Neurology. 2010 Apr 27;74(17):1340-5.

14. Zinkstok SM, Engelter ST, Gensicke H, et al. Safety of thrombolysis in stroke mimics: results from a multicenter cohort study. Stroke. 2013 Apr;44(4):1080-4.

15. Tsivgoulis G, Zand R, Katsanos AH, et al. Safety of intravenous thrombolysis in stroke mimics: prospective 5-year study and comprehensive meta-analysis. Stroke. 2015 May;46(5):1281-7.

16. Scheitz JF, Gensicke H, Zinkstok SM, et al. Cohort profile: Thrombolysis in Ischemic Stroke Patients (TRISP): a multicentre research collaboration. BMJ open. 2018 Sep 17;8(9):e023265.

17. Abend NS, Beslow LA, Smith SE, et al. Seizures as a presenting symptom of acute arterial ischemic stroke in childhood. The Journal of pediatrics. 2011 Sep;159(3):479-83.

18. Gensicke H, Zinkstok SM, Roos YB, et al. IV thrombolysis and renal function. Neurology. 2013 Nov 12;81(20):1780-8.

19. Hacke W, Kaste M, Fieschi C, et al. Randomised double-blind placebo-controlled trial of thrombolytic therapy with intravenous alteplase in acute ischaemic stroke (ECASS II). Second European-Australasian Acute Stroke Study Investigators. Lancet (London, England). 1998 Oct 17;352(9136):1245-51.

20. Fisher RS, Cross JH, D'Souza C, et al. Instruction manual for the ILAE 2017 operational classification of seizure types. Epilepsia. 2017 Apr;58(4):531-42.

21. Hacke W, Kaste M, Bluhmki E, et al. Thrombolysis with Alteplase 3 to 4.5 Hours after Acute Ischemic Stroke. New England Journal of Medicine. 2008;359(13):1317-29.

22. Scheffer IE, Berkovic S, Capovilla G, et al. ILAE classification of the epilepsies: Position paper of the ILAE Commission for Classification and Terminology. Epilepsia. 2017 Apr;58(4):512-21.

23. Beghi E, Carpio A, Forsgren L, et al. Recommendation for a definition of acute symptomatic seizure. Epilepsia. 2010 Apr;51(4):671-5.

24. Scheitz JF, Turc G, Kujala L, et al. Intracerebral Hemorrhage and Outcome After .. irombolysis in Stroke Patients Using Selective Serotonin-Reuptake Inhibitors. Stroke. 2017 Dec;48(12):3239-44.

25. van Buuren S, Groothuis-Oudshoorn K. mice: Multivariate Imputation by Chained Equations in R. 2011. 2011 2011-12-12;45(3):67.

26. lacus S, King G, Porro G. Causal Inference without Balance Checking: Coarsened Exact Matching. Political Analysis. 2012;20(01):1-24.

27. Hernan MA, Robins JM. Estimating causal effects from epidemiological data. Journal of epidemiology and community health. 2006 Jul;60(7):578-86. 
28. Austin PC, Stuart EA. Moving towards best practice when using inverse probability of treatment weighting (IPTW) using the propensity score to estimate causal treatment effects in observational studies. Statistics in medicine. 2015 Dec 10;34(28):3661-79.

29. Robins JM, Hernan MA, Brumback B. Marginal structural models and causal inference in epidemiology. Epidemiology (Cambridge, Mass). 2000 Sep;11(5):550-60.

30. $\mathrm{Xu} \mathrm{Y}$, Hackett ML, Chalmers J, et al. Frequency, determinants, and effects of early seizures after thrombolysis for acute ischemic stroke: The ENCHANTED trial. Neurology Clinical practice. 2017;7(4):324-32.

31. Alvarez V, Rossetti AO, Papavasileiou V, Michel P. Acute seizures in acute ischemic stroke: does thrombolysis have a role to play? Journal of neurology. 2013 Jan;260(1):55-61.

32. Jung $S$, Schindler K, Findling $O$, et al. Adverse effect of early epileptic seizures in patients receiving endovascular therapy for acute stroke. Stroke. 2012 Jun;43(6):1584-90.

33. Rodan LH, Aviv RI, Sahlas DJ, Murray BJ, Gladstone JP, Gladstone DJ. Seizures during stroke thrombolysis heralding dramatic neurologic recovery. Neurology. 2006 Dec 12;67(11):2048-9.

34. Adams HP, Jr., Davis PH, Leira EC, et al. Baseline NIH Stroke Scale score strongly predicts outcome after stroke: A report of the Trial of Org 10172 in Acute Stroke Treatment (TOAST). Neurology. 1999 Jul 13;53(1):126-31.

35. Ahmed N, Davalos A, Eriksson N, et al. Association of admission blood glucose and outcome in patients treated with intravenous thrombolysis: results from the Safe Implementation of Treatments in Stroke International Stroke Thrombolysis Register (SITSISTR). Archives of neurology. 2010 Sep;67(9):1123-30.

36. Hankey GJ, Spiesser J, Hakimi Z, Bego G, Carita P, Gabriel S. Rate, degree, and predictors of recovery from disability following ischemic stroke. Neurology. 2007 May 8;68(19):1583-7.

37. Gensicke H, Strbian D, Zinkstok SM, et al. Intravenous Thrombolysis in Patients Dependent on the Daily Help of Others Before Stroke. Stroke. 2016 Feb;47(2):450-6.

38. Huang CW, Saposnik G, Fang J, Steven DA, Burneo JG. Influence of seizures on stroke outcomes: a large multicenter study. Neurology. 2014 Mar 4;82(9):768-76.

39. Szaflarski JP, Rackley AY, Kleindorfer DO, et al. Incidence of seizures in the acute rilase of stroke: a population-based study. Epilepsia. 2008 Jun;49(6):974-81.

40. Hamidou B, Aboa-Eboule C, Durier J, et al. Prognostic value of early epileptic seizures on mortality and functional disability in acute stroke: the Dijon Stroke Registry (1985-2010). Journal of neurology. 2013 Apr;260(4):1043-51.

41. Procaccianti G, Zaniboni A, Rondelli F, Crisci M, Sacquegna T. Seizures in Acute Stroke: Incidence, Risk Factors and Prognosis. Neuroepidemiology. 2012;39(1):45-50.

42. Roivainen R, Haapaniemi E, Putaala J, Kaste M, Tatlisumak T. Young adult ischaemic stroke related acute symptomatic and late seizures: risk factors. European journal of neurology. 2013 2013/09//;20(9):1247-55. 
43. Bladin CF, Alexandrov AV, Bellavance A, et al. Seizures after stroke: a prospective multicenter study. Archives of neurology. 2000 Nov;57(11):1617-22.

44. Shah S, Luby M, Poole K, et al. Screening with MRI for Accurate and Rapid Stroke Treatment: SMART. Neurology. 2015 Jun 16;84(24):2438-44.

45. Quenardelle V, Lauer-Ober V, Zinchenko I, et al. Stroke Mimics in a Stroke Care Pathway Based on MRI Screening. Cerebrovascular diseases (Basel, Switzerland). 2016;42(3-4):205-12.

46. de Los Rios la Rosa F, Khoury J, Kissela BM, et al. Eligibility for Intravenous Recombinant Tissue-Type Plasminogen Activator Within a Population: The Effect of the European Cooperative Acute Stroke Study (ECASS) III Trial. Stroke. 2012 Jun;43(6):1591-5. 


\section{Figure legends}

Figure 1. Odds ratio estimates with $95 \% \mathrm{CI}$ for $\mathrm{sICH}$, 3-month mortality, poor 3-month functional outcome (mRS $\geq 3$ ) and shift to a higher 3-month $\mathrm{mRS}$ in unadjusted logistic regression (dashed line), adjusted logistic regression, matched and weighted analyses (solid lines) in patients with versus without seizure at onset. 
Table 1. Baseline characteristics stratified by seizure at onset

\begin{tabular}{|c|c|c|c|c|c|}
\hline & $\begin{array}{l}\text { all patients } \\
(\mathrm{N}=10,074)\end{array}$ & $\begin{array}{c}\text { overall } \\
\text { missing } \\
\text { values rate }\end{array}$ & $\begin{array}{l}\text { seizure at onset } \\
\qquad(N=146)\end{array}$ & $\begin{array}{c}\text { no seizure at } \\
\text { onset } \\
(\mathrm{N}=9,928)\end{array}$ & p-value \\
\hline age ver, median (IQR) & $73(62-80)$ & $0 \%$ & $71.0(61-79)$ & $73(62-80)$ & 0.185 \\
\hline le, $N(\%)$ & $4,507 / 10,074(44.7)$ & $0 \%$ & 79/146 (54.1) & $4,428 / 9,928(44.6)$ & 0.027 \\
\hline NI/ASS or admission, median (IQR) & $9(5-16)$ & $0.4 \%$ & $13(7-21)$ & $9(5-15)$ & $<0.001$ \\
\hline qlucnce nn admission, mmol/l, median (IQR) & $6.6(5.8-8.0)$ & $2.6 \%$ & $7.1(6.1-8.5)$ & $6.6(5.8-8.0)$ & 0.016 \\
\hline eGFR on admission, $\mathrm{ml} / \mathrm{min} / 1.73 \mathrm{~m}^{2}$, mean (SD) & $73.8(22.8)$ & $10.0 \%$ & $75.2(25.4)$ & $73.8(22.7)$ & 0.479 \\
\hline on et-to-treatment time, minutes, median (IQR) & $135(95-180)$ & $8.4 \%$ & $133.5(93.5-179.5)$ & $135(95-180)$ & 0.990 \\
\hline prior antithrombotic drug treatment, $\mathrm{N}(\%)$ & & $2.8 \%$ & & & 0.774 \\
\hline no an+ irombotic treatment & $5,392 / 9,789(55.1)$ & & $79 / 145(54.5)$ & $5,313 / 9,644(55.1)$ & \\
\hline platelets & $3,951 / 9,789(40.4)$ & & $61 / 145(42.1)$ & $3,890 / 9,644(40.3)$ & \\
\hline anticoagulants \pm antiplatelets & $446 / 9,789(4.4)$ & & $5 / 145(3.4)$ & $441 / 9,644(4.6)$ & \\
\hline IVT followed by EVT, N (\%) & $1,034 / 10,074(10.3)$ & $0 \%$ & $15 / 146(10.3)$ & $1,019 / 9,928(10.3)$ & 1.000 \\
\hline ke, N (\%) & $1,666 / 10,011(16.6)$ & $0.6 \%$ & $37 / 144(25.7)$ & $1,629 / 9,867(16.5)$ & 0.005 \\
\hline hyp sion, $N(\%)$ & $6,866 / 10,035(68.4)$ & $0.4 \%$ & $100 / 144(69.4)$ & $6,766 / 9,891(68.4)$ & 0.860 \\
\hline hyperchr lesterolemia, N (\%) & $4,100 / 9,983(41.1)$ & $0.9 \%$ & $57 / 143(39.9)$ & $4,043 / 9,840(41.1)$ & 0.833 \\
\hline diabetes mellitus, N (\%) & $1,935 / 10,029(19.3)$ & $0.4 \%$ & $25 / 145(17.2)$ & $1,910 / 9,884(19.3)$ & 0.600 \\
\hline atrial fibrillation, N (\%) & $2,759 / 9,914(27.8)$ & $1.6 \%$ & $32 / 144(22.2)$ & $2,727 / 9,770(27.9)$ & 0.156 \\
\hline co $r$ disease, $N(\%)$ & $1,891 / 10,004(18.9)$ & $0.7 \%$ & $22 / 145(15.2)$ & $1,869 / 9,859(19.0)$ & 0.294 \\
\hline priur fun tional dependence (mRS $\geq 3$ ), N (\%) & $653 / 8,448(7.7)$ & $16.1 \% *$ & $21 / 128(16.4)$ & $632 / 8,320(7.6)$ & $<0.001$ \\
\hline earlv hypodensity on initial CT scan, N (\%) & $1,298 / 4,740(27.4)$ & $53.0 \%{ }^{\dagger}$ & $22 / 122(18.0)$ & $1,276 / 4,618(27.6)$ & 0.019 \\
\hline fina. ...gnosis of stroke mimic, N (\%) & $259 / 9,871(2.6)$ & $2.0 \%$ & $57 / 146(39.0)$ & $202 / 9,725(2.1)$ & $<0.001$ \\
\hline
\end{tabular}

* iriormat on on prior functional dependence was not collected in the center Heidelberg

${ }^{\dagger}$ So. _enters predominantly used MRI for initial imaging evaluation. In the centers that used CT, information on early hypodensity was available only in a cubset of patients (variable added post hoc) 
B. Analyses for the association of seizure at onset with the outcome measures accounting for confounders

\begin{tabular}{|c|c|c|c|c|c|c|}
\hline - & \multicolumn{2}{|c|}{ adjusted logistic regression* } & \multicolumn{2}{|c|}{ matched analysis $^{\dagger}$} & \multicolumn{2}{|c|}{ weighted analysis ${ }^{\ddagger}$} \\
\hline & $\begin{array}{c}\text { OR } \\
{[95 \% \mathrm{Cl}]}\end{array}$ & p-value & $\begin{array}{c}\text { OR } \\
{[95 \% \mathrm{Cl}]}\end{array}$ & p-value & $\begin{array}{c}\text { OR } \\
{[95 \% \mathrm{Cl}]}\end{array}$ & p-value \\
\hline SICH (ECASS-II) & $\begin{array}{c}0.52 \\
{[0.13,2.16]}\end{array}$ & 0.37 & $\begin{array}{c}0.68 \\
{[0.15,3.03]}\end{array}$ & 0.61 & $\begin{array}{c}0.95 \\
{[0.39,2.32]}\end{array}$ & 0.97 \\
\hline 3-r.nonth nortality & $\begin{array}{c}0.98 \\
{[0.50,1.92]}\end{array}$ & 0.95 & $\begin{array}{c}1.13 \\
{[0.55,2.33]}\end{array}$ & 0.73 & $\begin{array}{c}1.17 \\
{[0.73,1.88]}\end{array}$ & 0.83 \\
\hline $\begin{array}{l}\text { month functional outcome } \\
\text { (dichotomized } \mathrm{mRS} \geq 3 \text { ) }\end{array}$ & $\begin{array}{c}0.78 \\
{[0.45,1.32]}\end{array}$ & 0.35 & $\begin{array}{c}0.75 \\
{[0.43,1.32]}\end{array}$ & 0.32 & $\begin{array}{c}0.87 \\
{[0.57,1.34]}\end{array}$ & 0.83 \\
\hline ordinal 3-month mRS & $\begin{array}{c}0.78 \\
{[0.52,1.16]}\end{array}$ & 0.22 & $\begin{array}{c}0.45 \\
{[0.10,2.06]}\end{array}$ & 0.3 & $\begin{array}{c}1.00 \\
{[0.66,1.52]}\end{array}$ & 1.00 \\
\hline
\end{tabular}

* adjustment for age, sex, NIHSS on admission, prior antithrombotic treatment, glucose and eGFR on admission, EVT in addition to IVT, onset-totreatment time, prior dependence and prior stroke

${ }^{\dagger}$ matching for age, sex, NIHSS, glucose and eGFR on admission, prior stroke and final diagnosis

${ }^{\ddagger}$ weighting with age, sex, NIHSS on admission, prior antithrombotic treatment, glucose and eGFR on admission, EVT in addition to IVT, onset-totreatment time, prior stroke and final diagnosis 


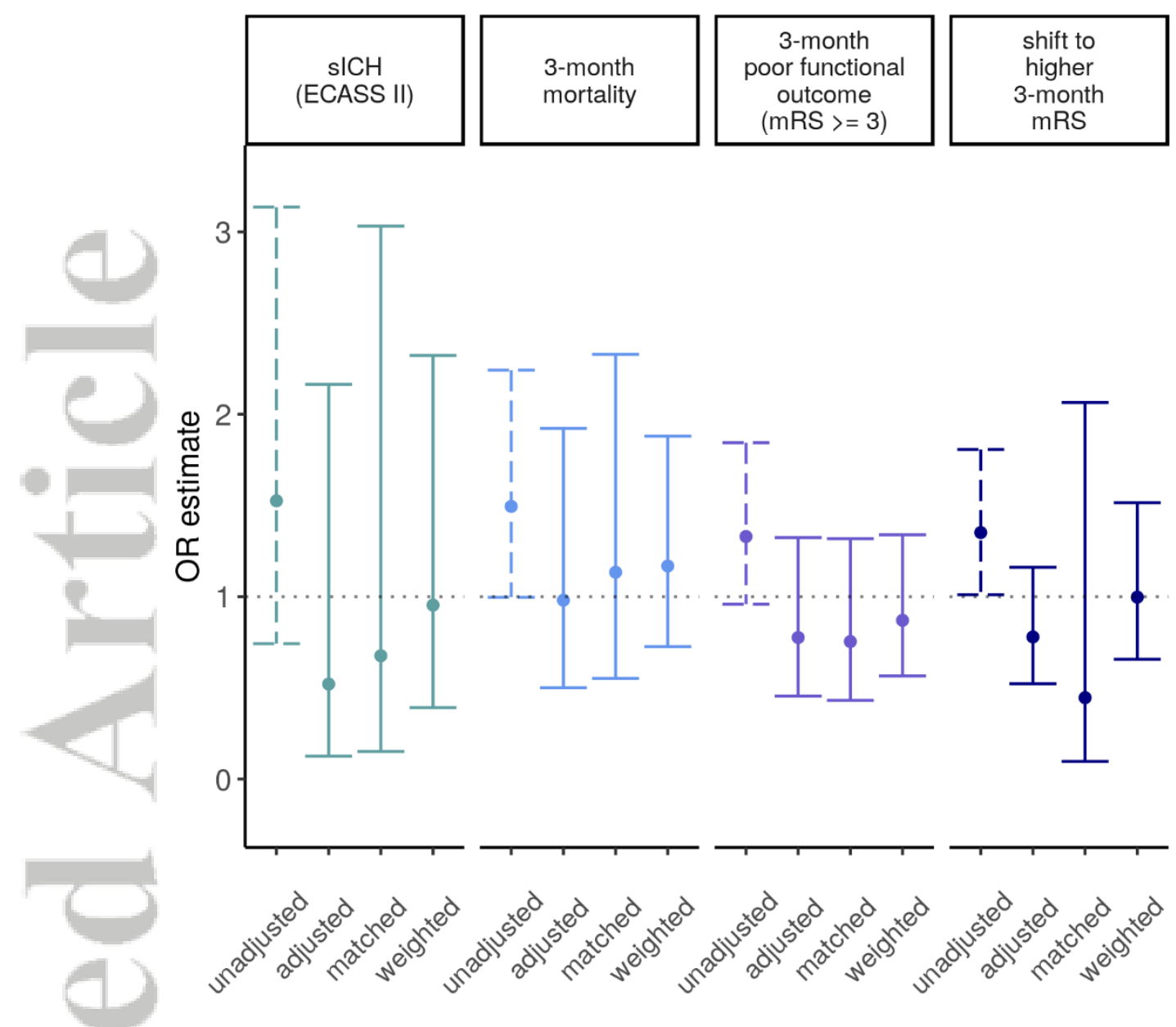

This article is protected by copyright. All rights reserved. 